\title{
Paper
}

\section{Mechanical Treatment of Silica Powder}

\author{
TRAN Thi Thu Hien ${ }^{1,2}$, Takashi SHIRAI ${ }^{1}$ and Masayoshi FUJI ${ }^{1}$ \\ ${ }^{1}$ Advanced Ceramics Research Laboratory, Nagoya Institute of Technology, 3-101-1 Honmachi, Tajimi 507-0033, Japan. \\ ${ }^{2}$ School of Materials Science and Technology, Hanoi University of Science and Technology (Dai hoc Bach khoa Hanoi), $1^{\text {st }}$ Dai Co Viet Street, Hanoi, Vietnam.
}

Received March 26, 2015; Revised June 10, 2015; Accepted June 24, 2015

\begin{abstract}
An amorphous silica powder (A2) was mechanically treated using a planetary type mill. Four different rotation speeds $(50,100,200,300 \mathrm{rpm})$, milling times $(15,30,60 \mathrm{~min})$ and ball sizes $(01,05,10 \mathrm{~mm})$ were used. The structure and morphology of the powder did not change by milling. The effect of milling conditions on particle size distribution and specific surface area was investigated. By the milling treatment the original particle size of the powder was not effectively reduced. Rotation speeds of more than $200 \mathrm{rpm}$ and prolonged milling causes the formation of a broad range of particles of larger sizes. The surface activity of raw and treated powders was measured as the amounts of dissolved $\mathrm{Si}^{4+}$ ion into ammonia solution. The $10 \mathrm{~mm}$ ball size was the most effective for 200 and $50 \mathrm{rpm}$ rotation speeds almost independently of the milling time. Comparison of the effect of milling conditions on particle features of this powder with previously investigated powder is also discussed. The findings indicate that from a technical and economical point of view each powder has its own optimum milling condition, which will be sufficient to considerably increase the specific surface activity of the original amorphous powder making it suitable for different applications in non-firing ceramics processing.
\end{abstract}

\section{KEY WORDS}

Silica powder, mechanical treatment, surface activities, modifications

\section{Introduction}

Silica $\left(\mathrm{SiO}_{2}\right)$ is one of the most abundant minerals on the earth's surface and is an important technological material. It can have application in numerous areas especially in glass. The functionalization of the silica particle surface can be used to enhance and/or control the overall properties of the particles for desired applications. The nature of the functional groups on the particle surface usually plays a critical role in the surface properties of particles such as hydrophobicity and chemical reactivity. There has been increasing interest in the synthesis and application of oxide materials whose surface is modified by organic, inorganic and metal-complex compounds ${ }^{1-3)}$. The significant property of these materials is that they combine the physical properties of solids and the chemical behavior of surface compounds.

The surface silicon atoms tend to have a complete tetrahedral configuration and in an aqueous medium their free valence becomes saturated with hydroxyl groups (surface $\mathrm{OH}$ groups: isolated, germinal, H-bonded), siloxane groups ( $\mathrm{Si}-\mathrm{O}-\mathrm{Si}$ or bridges with oxygen atoms on the surface $)^{4,5)}$. Deposition of different compounds on the surface of $\mathrm{SiO}$ based materials

* Corresponding author, E-mail: shirai@nitech.ac.jp is usually achieved by adsorption, the adsorption efficiency increases proportionally to the quantity of surface active sites. Many investigations have been reported on surface treatment and characterization of silica powders ${ }^{1,5-8)}$.

Recently, Apiluck et al. ${ }^{9}$ have proposed a novel ceramic fabrication method without firing and/or debinding process, in order to reduce the cost and energy for post-forming processes. In this method, the surface of a raw powder is mechanically activated using a planetary ball-mill. In the present work, the surface of an amorphous silica powder was activated by mechanical treatment for further applications in synthesis of non-firing ceramic. A variety of milling conditions were carried out combining different rotation speeds, milling times and ball sizes. BET, PSD, ICP, SEM were used for characterization. The optimum milling conditions to activate the surface of the present powder are compared with the results obtained previously for another amorphous silica powder.

\section{Experimental Procedure}

The silica powder IFX (A2) used in the present investigation was supplied by TOSO Ltd., Japan.

A FRITSCH-Pulverisette 5-Germany type laboratory planetary mill was used to mill the silica under air at room temperature. Raw 

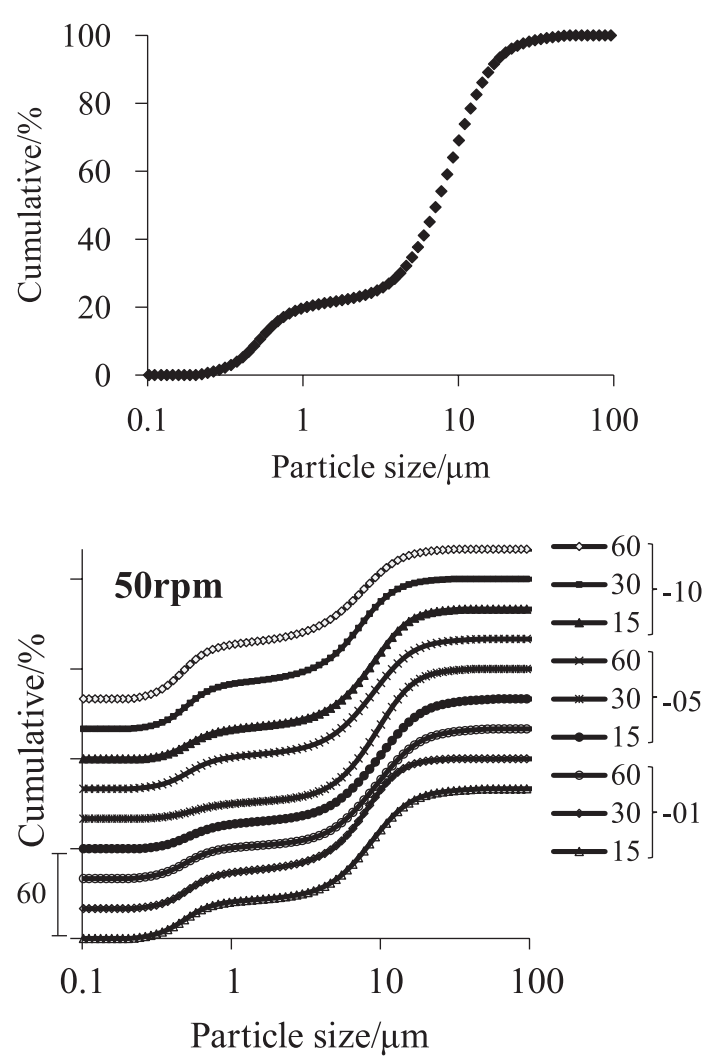
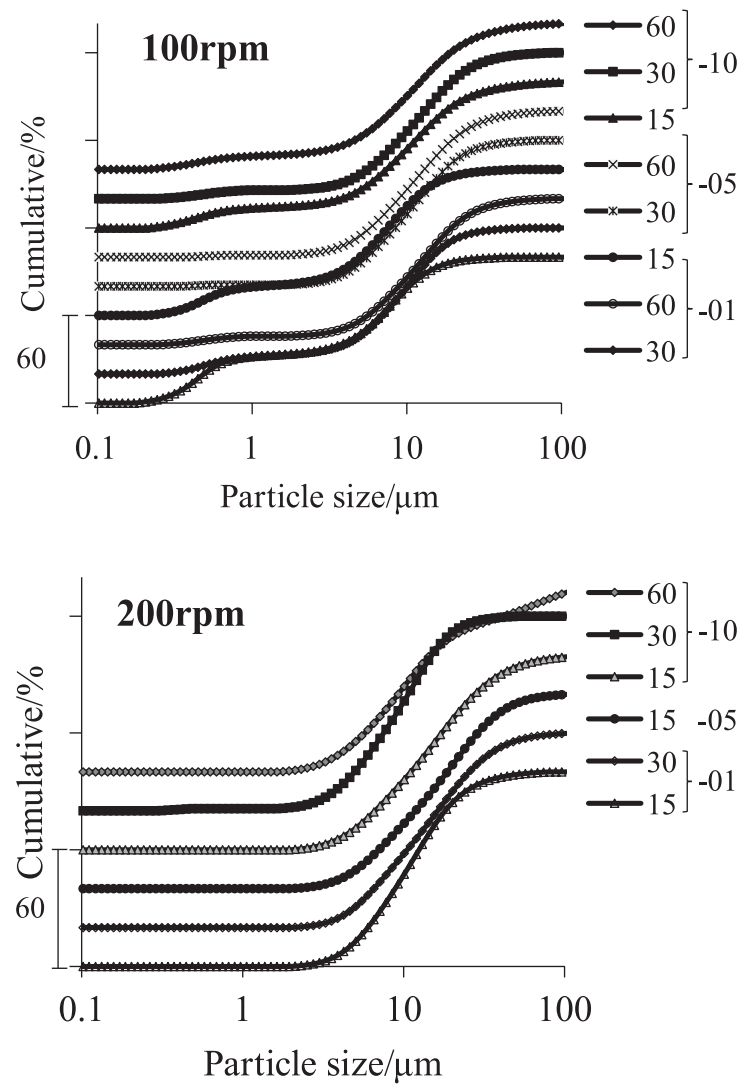

Fig. 1 Particle size distribution of raw and milled powder at every milling condition. 60,30 and 15 indicate milling time in minutes. 01 , 05 and 10 correspond to the ball size in $\mathrm{mm}$. Raw powder particle size was $10 \mu \mathrm{m}$. By increasing the speed and milling time a peak of small size particles disappeared, probably due to agglomeration of smaller particles.

silica powder samples were milled in a $500 \mathrm{ml}$ zirconia pot using zirconia balls for periods of 15,30 and 60 minutes. Rotation speeds of 50,100, 200 and $300 \mathrm{rpm}$ were applied. Three different ball sizes; 01,05 and $10 \mathrm{~mm}$ were used. For all the experiments $25 \mathrm{~g}$ of raw powder was used with a weight ratio to the zirconia ball of 1:4.

Particle size distribution (PSD) measurements were carried out on a MICROTRAC Particle Size Analyzer after ultrasonically dispersing the powders in distilled water for 20 minutes.

Particle morphology observation was carried out using Scanning Electron Microscopy (SEM) with JSM-7000F type equipment. Powders were put into carbon paste and dried in vacuum dryer for 24 hours before observation.

BET method was used to measure specific surface area (SSA) of the powders from adsorption-desorption of $\mathrm{N}_{2}$ at $77.3 \mathrm{~K}$ with BELSORP Max type equipment.

The quantitative dissolved loads of cation $\mathrm{Si}^{4+}$ concentration were estimated by using ICP technique (Inductively Couple Plasma)-SPS-7800 type. The sample for ICP analysis was prepared by dissolving $1 \mathrm{~g}$ powder with $100 \mathrm{ml}$ of ammonia solution $\left(\mathrm{NH}_{3}\right.$ $3 \mathrm{M})$. The solutions were kept shaking continuously at atmosphere and room temperature $\left(25^{\circ} \mathrm{C}\right)$ for 2 hours (AS ONE-Shaking baths SB20) and the slurries were separated and centrifuged (KOKUSAN type). The upper portion was filtered with 0.2 micron size filter to remove the remaining particles and the solution was used for ICP measurement.

\section{Results and Discussions}

The changes in the particle size distribution (PSD) of the raw and treated powder under different milling conditions are presented in Fig. 1. Milling conditions are indicated in the figures and the meaning of the nomenclature is explained in the captions. The powder presents a bimodal particle size distribution composed of $70 \%$ of larger particles in the size range 4 and $22 \mu \mathrm{m}$ and $15 \%$ of smaller particles in the range $0.3-0.8 \mu \mathrm{m}$. The particle size distributions of milled powders at rotation speed of $50 \mathrm{rpm}$ are similar to that of raw powder. The peak of small size particles disappeared at high rotation speed of $200 \mathrm{rpm}$. At $100 \mathrm{rpm}$ prolonged milling causes changes in the range of particles of smaller sizes. This observation may be explained as due to the agglomeration of smaller particles as discussed in $^{10)}$.

As seen in Fig. 2 the shape and size of the original particles did not change by the milling modifications. This image is in agreement with the particle size distribution shown in Fig. 1 for these milling conditions. This feature was also observed for previously investigated powders (A1) ${ }^{10)}$.

Fig. 3 shows specific surface area values of raw and milled 


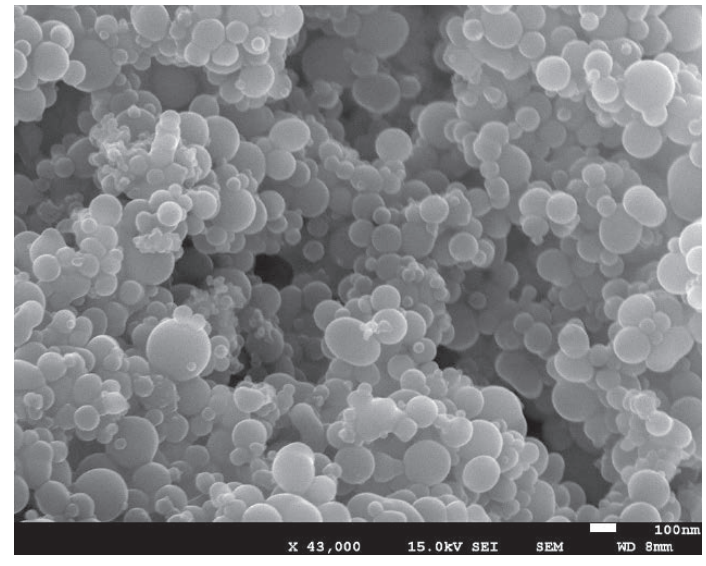

IFX (A2)-Raw

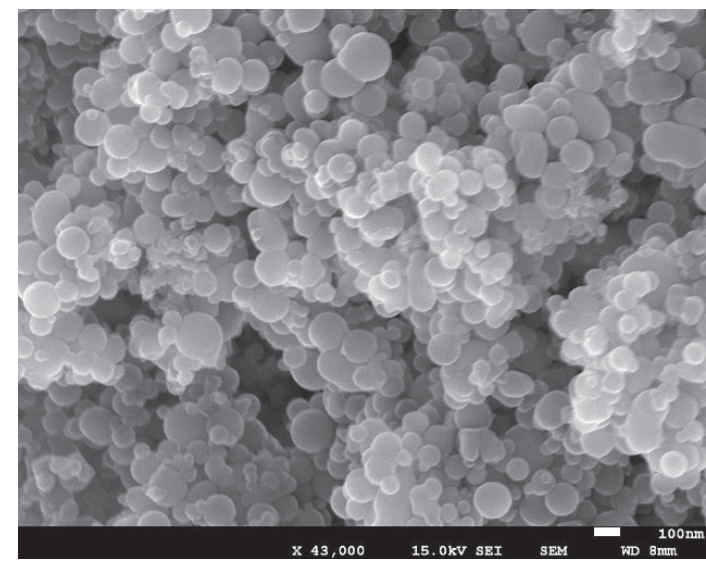

IFX (A2)-100-15-01

Fig. 2 Morphology of raw and mechanically treated (rotation $100 \mathrm{rpm}$-milling time $15 \mathrm{~min}$ - ball size $01 \mathrm{~mm}$ ) powders observed using SEM spectroscopy. The observed particle sizes are in agreement with the measured particle size distribution for these treatment conditions. The shape did not change after milling process.
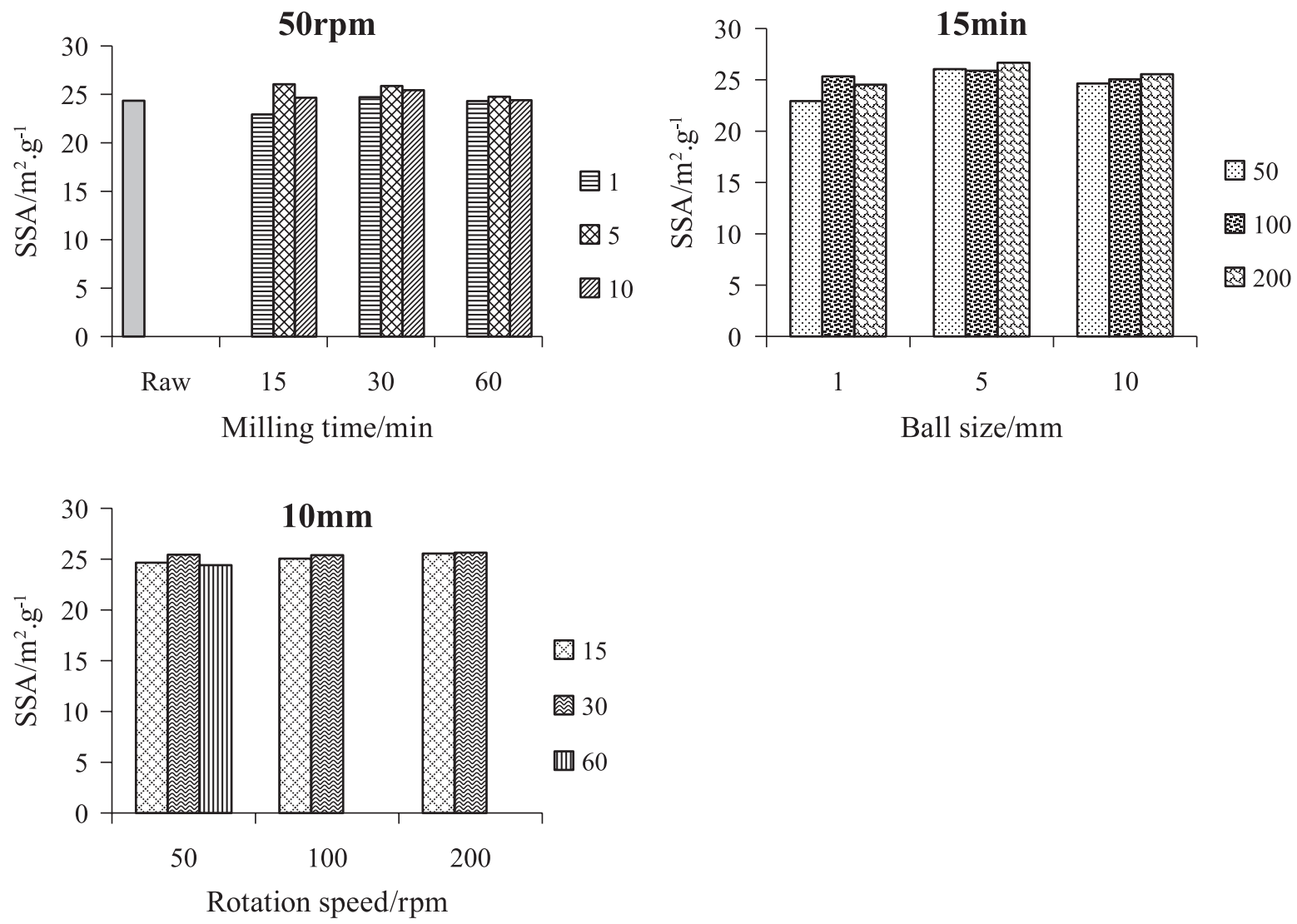

Fig. 3 Specific surface area values of raw and treated powders. Fixed parameters are indicated in each case. 60, 30 and 15 indicate milling time in minutes. 01,05 and 10 correspond to the ball size in mm. 50,100 and 200 are rotation speeds. There is a very small increased of SSA when the milling speed, milling time, ball size is increased to $100 \mathrm{rpm}, 30 \mathrm{~min}$ and $5 \mathrm{~mm}$, respectively. SSA decreased when prolonged milling speed, milling time or big ball size.

amorphous silica A2 powders. 1, 5 and 10 indicate ball size in $\mathrm{mm} .15,30$ and 60 correspond to milling time in min. 50, 100 and $200 \mathrm{rpm}$ are rotation speeds. The fixed parameter is also shown. The SSA values of all powders were in the range of $23-26 \mathrm{~m}^{2} / \mathrm{g}$ (within $11.5 \%$ difference). The behavior of small change in SSA was also reported in the modification of another powder ${ }^{10)}$. SSA increased a little at a rotation speed of $100 \mathrm{rpm}$, milling time of
$30 \mathrm{~min}$ and ball size of $5 \mathrm{~mm}$. When prolonged milling time, high milling speed and large ball size corresponding to $60 \mathrm{~min}$, $200 \mathrm{rpm}$ and $10 \mathrm{~mm}$ are used, re-agglomeration occurs and the SSA decreases. From these results, long time milling is not only unsuitable for economical reasons but also because the particle features are not appropriate for application. The particle size distribution and specific surface area of the original power hardly 
changed by the milling treatment but prolonged milling time, high milling speed and large ball size caused detrimental agglomeration of small particles.

These features were also observed for a previously investigated powder (A1) but milled conditions are different. SSA for the A1 studied powder also increase hardly but at higher rotation speed (300 rpm) and higher milling time (60 min) compare to the present A2 powder. The powder A1 is agglomerated stated so that it needs more energy to break the aggregated particles in order to increase SSA. In the case of A2 powder, original particles are well dispersed, it enhances less used energy to separate the aggregated particles. In generally, at higher rotation speed or milling time, the harder agglomerated particles are broken to induce increasing of SSA but for each powder has its own curtain milling conditions ${ }^{10)}$.

As we proposed in an earlier communication, the mechanical process induces surface damage resulting in broken bonds and generation of surface silicon ions ${ }^{10)}$. The surface activity of raw and treated powders at various conditions was measured as the amounts of dissolved $\mathrm{Si}^{4+}$ ion into ammonia solution after 2 hours shaking time. Surface area normalized dissolved silicon ion values for raw and treated silica powder is shown in Fig. 4. The specific surface activity increased by the mechanical treatment at every tested condition. For this powder the highest ball size, $10 \mathrm{~mm}$ was the most effective for 100 and $200 \mathrm{rpm}$ rotation speeds almost independently of the milling time. At $300 \mathrm{rpm}$ of milling speed, surface activities decreased because of re-agglomeration of small particles. According to these results, from an economical point of view 15 min milling time at $200 \mathrm{rpm}$ with a $10 \mathrm{~mm}$ ball will be sufficient to considerably increase the specific surface activity of this powder (A2). In the case of A1 investigated powder, the optimum conditions were different in ball size as shown $15 \mathrm{~min}$ milling time at $200 \mathrm{rpm}$ with a $05 \mathrm{~mm}$. As results in dissolved $\mathrm{Si}^{4+}$ in ammonia solution, the ball size is the most effective factor to activate the surface activity but each powder gives different results in ball sizes. It may possible the original surface state, surface features or further production method of the powders, the optimum conditions for sufficiently increasing the surface activity of the powder investigated in the present study are different from the one previously studied indicating that the effect of milling on the surface activity may be different for each powder and the conditions can not be generalized. The difference of original surface state for each powder will be shown in further publication.

\section{Summary and Conclusions}

By planetary milling process the particle size and specific surface activity of amorphous silica powder were successfully modified. Milling at different rotation speeds, milling times and ball sizes was applied and the results can be summarized as follows:

- The particles morphology of the original powder did not change by the milling treatment. This feature was also

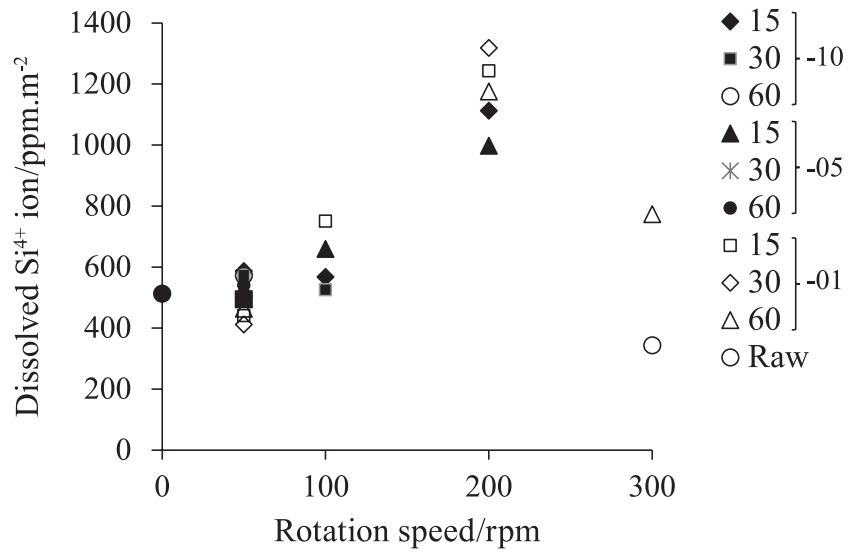

Fig. 4 Surface area normalized dissolved $\mathrm{Si}^{4+}$ ion values for raw and treated amorphous silica powder at various milling conditions. 60 , 30 and 15 indicate milling time in minutes. 01,05 and 10 correspond to the ball size in $\mathrm{mm}$. The specific surface activity of the powder could be successfully increased by the mechanical treatment. The maximum activities are seen at $200 \mathrm{rpm}$ of rotation speed.

observed for a previously investigated powder.

- The particle size distribution and specific surface area of the original power hardly changed by the milling treatment but prolonged milling time, high milling speed and large ball size caused detrimental agglomeration of small particles. These features were also observed for a previously investigated powder.

- According to the results of the present work, from a technical and economical point of view $15 \mathrm{~min}$ milling time at $200 \mathrm{rpm}$ rotation speed with a $10 \mathrm{~mm}$ ball size will be sufficient to increase the specific surface activity of the powder making it suitable for different applications in non-firing ceramics processing.

By planetary milling process the particle size and specific surface activity of amorphous silica powder were successfully modified.

\section{Acknowledgements}

The authors are in debt to the Chubu Science and Technology Center for financial support to Tran Thi Thu Hien during the course of this research. Appreciation is also extended to Dr. Chanel Ishizaki for useful discussions. We also would like to thank TOSO Company for powder supply and research collaboration.

\section{References}

1) L. Teokcharov, D. Simeonov, I. Uzunov, D. Klissurski: "Mechanochemical modification of silica", J. Materials Sci. Let., 11 (1992) 1180-1182.

2) W. E. E. Ston, G. M. S. El Shafei, J. Sanz, S. A. Selim: "Association of soluble aluminum ionic species with a silica-gel surface A solid-state NMR study”, J. Phys. Chem., 97 (1993) 10127-10132.

3) B. A. Fleming: "Kinetics of reaction between silicic acid 
amorphous silica surface in $\mathrm{NaCl}$ solutions", J. Colloid and Inter. Sci., 110 (1986) 40-64.

4) L. T. Zhuravlev: "Surface characterization of amorphous silica-a review of work from the former USSA, Colloids and Surfaces A: Physicochemical and Engineering”, Aspects, 74 (1993) 71-90

5) L. T. Zhuravlev: The surface chemistry of amorphous silica, Colloids and Surfaces A: Physicochemical and Engineering Aspects, 173 (2000) 1-38.

6) I. A. Vorsina, T. F. Grigorieva, A. P. Barinova, N. Z. Lyakhov: “Chemistry for sustainable development”, 6 (1998) 236-248.
7) P. F. Holt, D. T. King: "The chemistry of silica surfaces", J. Chem. Soc., (1955) 773-779.

8) T. T. Trinh, A. P. J. Jansen, R. A. van Santen: "Mechanism of oligomerization reactions of silica", J. Phys. Chem., B110 (2006) 23099-23106.

9) A. Elad-ua, T. Shirai, T. Kato, K. Orito, H. Watanabe, M. Fuji, N. Takahashi: "Novel fabrication route for porous ceramics using waste materials by non-firing process", J. Cer. Soc. Jpn., 118 (2010) 745-748.

10) T. T. T. Hien, T. Shirai, M. Fuji: "Mechanical modifications of silica powders”, J. Ceram. Soc. Jpn., 120 (2012) 429-435. 